\title{
EFICIENCIA EN EL USO DEL AGUA POR LAS PLANTAS
}

\author{
Hipólito Medrano, Josefina Bota, Josep Cifre, Jaume Flexas, Miquel Ribas-Carbó y \\ Javier Gulías \\ Grup de Biologia de les plantes en condicions mediterrànies \\ Departament de Biologia \\ Universitat de les Illes Balears- IMEDEA
}

\section{RESUMEN}

La disponibilidad de agua es el principal factor limitante de la producción agrícola y ganadera en ambientes de clima mediterráneo. Limitación que, ante las previsiones de Cambio Climático Global realizadas por organismo internacionales, serán mucho mayores en los próximos años. En este escenario, la eficiencia en el uso de los recursos hídricos debe ser un aspecto transversal de las políticas públicas que debe, por tanto, ser afrontado desde diversos puntos de vista. En este sentido, uno de los temas claves a considerar es la eficiencia con la que las plantas usan el agua. El objetivo de este trabajo es hacer una revisión de los diferentes aspectos relacionados con este tema, considerando las diferentes escalas a las que se estudia la eficiencia en el uso del agua por las plantas (EUA), desde la hoja hasta el cultivo o el ecosistema. Así, se abordan las dificultades técnicas que existen para medir, de una forma precisa, la EUA de un cultivo o de un ecosistema, la importancia del ambiente y de las prácticas agronómicas como determinantes de la EUA, la diversidad genética inter e intraespecífica, y las implicaciones prácticas de estos factores a la hora de incrementar la EUA.

Palabras clave: Agricultura, Clima Mediterráneo, Déficit hídrico, Eficiencia en el uso del agua, Fotosíntesis, Transpiración.

\begin{abstract}
Water availability is the most important limiting factor in plant and animal production under Mediterranean conditions. In a Global Climate Change scenario, this limitation will be even greater in the following years, according to the International Institutions predictions. In this sense, the efficiency of water resources utilization should be a key point of public policies that must be discussed from different points of view. One of these is Plant Water Use Efficiency (WUE). The objective of this paper is to review the different aspects related to WUE, considering the approach levels, from leaf to crop or ecosystem. The technical difficulties to measure accurately WUE at crop or ecosystem level, the environment and agronomical practices importance in WUE determination, the
\end{abstract}


inter and intraspecific plant genetic diversity, as well as the practical implications of each factor to increase WUE are discussed.

Key words: Agricultura, Mediterranean Climate, Photosynthesis, Transpiration, Water deficit, Water Use Efficiency.

\section{Introducción}

El agua es un recurso natural imprescindible, del que cada vez se requiere un mayor consumo en las poblaciones urbanas y que cada vez resulta más escaso. En la cultura mediterránea, la dificultad de abastecimiento ha generado formas de abastecimiento y consumo que primaba la economía y previsión, toda una cultura de la economía del agua que en cierta forma languidece frente a la preponderancia del modo de vida urbano respecto al rural. Sin embargo, las limitaciones de disponibilidad están afectando cada vez más a toda la población por lo que la economía en la red de abastecimiento, distribución, y sobretodo en los hábitos de consumo está cada vez más presente en nuestra sociedad.

Esta situación hace que el gasto de agua en la agricultura, que supone entre el 50 y el $80 \%$ del agua disponible (tabla 1), se mire como un «exceso» desde ciertos ámbitos, ignorando que ese consumo se dedica a producir los alimentos que la sociedad urbana necesita consumir y que, por tanto, acaba siendo también una necesidad de la población en general.

En este sentido, la economía en el uso del agua en sistemas agrícolas constituye una prioridad fundamental dada su fuerte repercusión en el monto total de agua utilizada. Sin embargo, la producción agrícola, por exigencias de la economía de mercado, necesita cada vez más asegurar unos rendimientos mínimos para ser una actividad viable, y el riego se hace cada vez más imprescindible para obtener una producción más regular y predecible

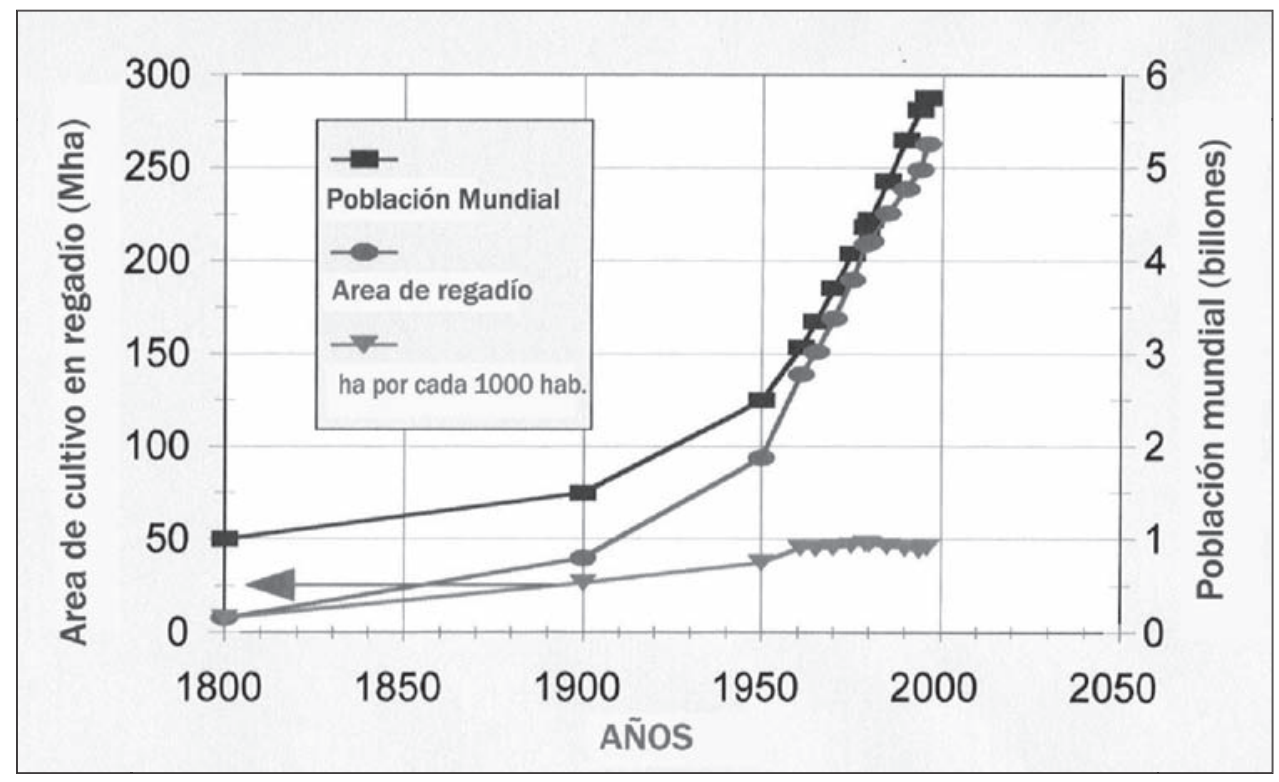

Figura 1. Tendencias mundiales en la superficie cultivada en regadío y población. 
de los cultivos. De esta forma, en el mediterráneo, incluso en cultivos tradicionalmente de «secano», como el olivo, el almendro, la vid etc., se implanta el riego como una necesidad para la rentabilidad de las explotaciones agrícolas. En las últimas décadas, la producción agrícola mundial ha aumentado en paralelo a los aumentos de población sin apenas aumentar la superficie cultivada (probablemente muy cercana al máximo disponible). El incremento de producción global de alimentos viene totalmente ligado al incremento de la superficie regada, y esta tendencia «universal» se mantiene o se acentúa en los últimos años (figura 1).

Afortunadamente, cada vez hay más conocimientos y tecnologías disponibles que ayudan a incrementar la eficiencia en el uso del agua en la agricultura y así, en nuestro país, el incremento registrado en la superficie de regadío declarada en los últimos años se ha hecho compatible con una reducción global de la cantidad de agua utilizada por la agricultura. La explicación está en que en la mayor parte de las nuevas áreas de riego se ha establecido el riego localizado, por goteo o aspersión, sistemas mucho más eficientes, y en cultivos, que en buena parte tienen un consumo unitario mucho más bajo que los cultivos hortícolas.

Mejorar la producción vegetal es compatible con la economía del agua, pero esto requiere más conocimientos y tecnología que debemos desarrollar para hacer más sostenible la producción de alimentos y el abastecimiento de las poblaciones. En el esquema (figura 2) se reseñan un conjunto de vías, desde los incentivos institucionales hasta la implantación de riegos de alta precisión, que permiten incrementar sensiblemente la economía del agua en los sistemas agrícolas.

La contribución de nuestro grupo de investigación a este empeño se centra en el incremento de los conocimientos sobre la ecofisiología de eficiencia del uso del agua por las plantas, de las necesidades de agua en procesos claves para la producción como la fotosíntesis, y en delimitar hasta qué punto es posible incrementar la eficiencia en el uso del agua por las plantas. Esto exige conocer mucho mejor los procesos claves para la producción, el peso de las variaciones climáticas y edáficas sobre la eficiencia de estos procesos y también identificar especies y variedades con mayor eficiencia de cara a la obtención de nuevas variedades de mayor eficiencia en el uso del agua.

Tabla 1

USOS DEL AGUA EN DIFERENTES ZONAS DEL MUNDO (1990)

\begin{tabular}{|l|c|c|c|c|c|}
\hline & Agricultura \% & $\begin{array}{c}\text { Doméstico } \\
\%\end{array}$ & $\begin{array}{c}\text { Industria } \\
\%\end{array}$ & Total $\mathbf{K m}^{3} /$ /año & $\begin{array}{c}\text { Requerimientos } \\
\mathbf{m}^{3} / \text { habitante/año }\end{array}$ \\
\hline África & 88 & 7 & 5 & 144 & 245 \\
\hline Asia & 86 & 6 & 8 & 1531 & 519 \\
\hline Ex USSR & 65 & 7 & 28 & 358 & 1280 \\
\hline Europa & 33 & 13 & 54 & 359 & 713 \\
\hline América N y Central & 49 & 9 & 42 & 697 & 1861 \\
\hline Oceania & 34 & 64 & 2 & 23 & 905 \\
\hline Sudamérica & 59 & 19 & 23 & 133 & 478 \\
\hline Mundo & 69 & 8 & 23 & 3240 & 644 \\
\hline
\end{tabular}

Fuente: Worl Resources Institute, 1994. 
Figura 2

VÍAS DE ACTUACIÓN PARA INCREMENTAR LA EFICIENCIA EN EL USO DEL AGUA

\section{OPCIONES PARA INCREMENTAR LA EFICIENCIA EN EL USO DEL AGUA POR LAS PLANTAS}

1. Institucional: Educación y formación; Infraestructuras de captación y suministro, Incentivos legales y multas

2. Implantación de sistemas de cultivo y de riego para optimizar el control del agua aplicada.

3. Manejo del suelo orientado a incrementar la EUA (introducción de residuos, coberturas). Estrategias de ordenación de cultivos, rotaciones, marcos de plantación.

4. Introducción de especies y variedades de mayor EUA.

5. Aplicación de calendarios y dosis de riego orientados a incrementar la EUA. Riego deficitario controlado. Agricultura de la precisión

\section{Recursos hídricos y producción vegetal}

La producción de nueva biomasa en cualquier cultivo o comunidad vegetal está fuertemente determinada por la cantidad de agua disponible en el suelo. Esto resulta evidente a la simple observación del paisaje natural y lo es mucho más cuando se cuantifica la producción anual (cosecha o biomasa acumulada en $\mathrm{g} / \mathrm{ha}$ ) y el agua utilizada (en $\mathrm{m}^{3} / \mathrm{ha}$ ). Como muestra la figura 3a, esta relación se cumple cuando comparamos precipitación anual y producción en diferentes biomas, a pesar de la fuerte interferencia de otros factores limitantes como la temperatura, la disponibilidad de nutrientes o las horas de luz, así como de las dificultades de estimación de la producción de biomasa en determinados biomas, la relación resulta evidente a escala global. Cuando la comparación se hace para una única especie y en diferentes regímenes de disponibilidad hídrica, el ajuste de la producción a la disponibilidad de agua es muy superior, de forma que la cosecha queda totalmente determinada por el agua utilizada (figura 3b).

La razón está en que le proceso base de la producción de nueva biomasa (la fotosíntesis) y el del gasto de agua (transpiración) se producen a la vez, y la entrada del dióxido de carbono y la salida del agua utilizan la misma vía, los estomas en las hojas. Cuanto más abiertos están, mas fácilmente entra el $\mathrm{CO}_{2}$ pero también más rápidamente se escapa el agua. El precio, el agua gastada para la producción biomasa, es por tanto inevitable y alto. La producción de biomasa sin agua es imposible para las plantas. La cuestión está en conocer más sobre si se trata de un coste único, si varía con el tipo de planta, si depende de las condiciones ambientales, etc. Este tema ha sido motivo de estudio desde finales del siglo XIX y principios del XX por agrónomos que establecieron las necesidades de agua de diferentes cultivos y sus variaciones en función de las condiciones ambientales (tabla 2). Producir un gramo de biomasa supone gastar entre 100 y $1000 \mathrm{~g}$ de agua y el valor exacto depende tanto del tipo de planta como de las condiciones ambientales. La causa de que algunos cultivos presentan menores requerimientos hídricos por kilo de cosecha (caña de azúcar, maíz, sorgo) se esclareció en los años 60 por fisiólogos que demostraron que estas especies disponían de una vía de fotosíntesis de mayor rendimiento (fotosíntesis $\mathrm{C}_{4}$ ), y abrió planteamientos nuevos sobre la capacidad de mejorar la economía hídrica de las plantas aumentando la eficiencia de los procesos fotosintéticos. 
Tabla 2

EFICIENCIAS DE LA TRANSPIRACIÓN (EE ${ }_{B}$ g biomasa $\mathrm{kg}^{-1}$ agua) DE VARIOS CULTIVOS

\begin{tabular}{|c|c|c|}
\hline Especie & Núm. de observaciones & $\mathbf{E E}_{\mathbf{B}}$ \\
\hline \multicolumn{3}{|l|}{ C4 gramíneas } \\
\hline Mijo & 10 & 3,75 \\
\hline Sorgo & 10 & 3,29 \\
\hline Pasto del Sudán & 5 & 2,63 \\
\hline Maíz & 33 & 2,76 \\
\hline \multicolumn{3}{|l|}{ C3 gramíneas } \\
\hline Cebada & 12 & 1,93 \\
\hline Trigo & 37 & 1,87 \\
\hline Centeno & 6 & 1,58 \\
\hline Avena & 18 & 1,72 \\
\hline Arroz & 2 & 1,47 \\
\hline \multicolumn{3}{|l|}{ C3 cultivos } \\
\hline Colza & 1 & 1,62 \\
\hline Patata & 6 & 1,74 \\
\hline Algodón & 6 & 1,76 \\
\hline \multicolumn{3}{|l|}{ C3 leguminosas } \\
\hline Trébol rojo & 1 & 1,43 \\
\hline Arveja & 3 & 1,70 \\
\hline Trébol dulce & 2 & 1,37 \\
\hline Guisante & 2 & 1,34 \\
\hline Alfalfa & 13 & 1,33 \\
\hline
\end{tabular}

Fuente: Datos originales de Shantz \& Piemeisel (1927).

La comprensión de los procesos fisiológicos que determinan los flujos de agua en las plantas permite plantear la cuestión de la eficiencia en el uso del agua como un problema de control gastos (agua) respecto de los ingresos en que, en primer lugar, hay que tener en cuenta las variaciones de la disponibilidad y de la necesidad de agua. La disponibilidad de agua en el suelo depende de los ingresos (lluvia, nieve, corrientes subterráneas, riego), de la capacidad de almacenamiento del suelo (proporción de elementos grueso, potencia, y porosidad del suelo) y de la densidad y profundidad del sistema radicular de la planta, que determina el volumen de suelo utilizado respecto del total. Así, la extensión del sistema radicular es un factor determinante de la disponibilidad real de agua, «el depósito de reserva» 
FIGURA 3a. Relación entre la productividad vegetal en diferentes ecosistemas y biomas (Kg de biomasa seca $\mathrm{m}^{-2}$ año $\mathrm{o}^{-1}$ y la precipitación anual $\left(\mathrm{mm} \mathrm{año}^{-1}\right)$

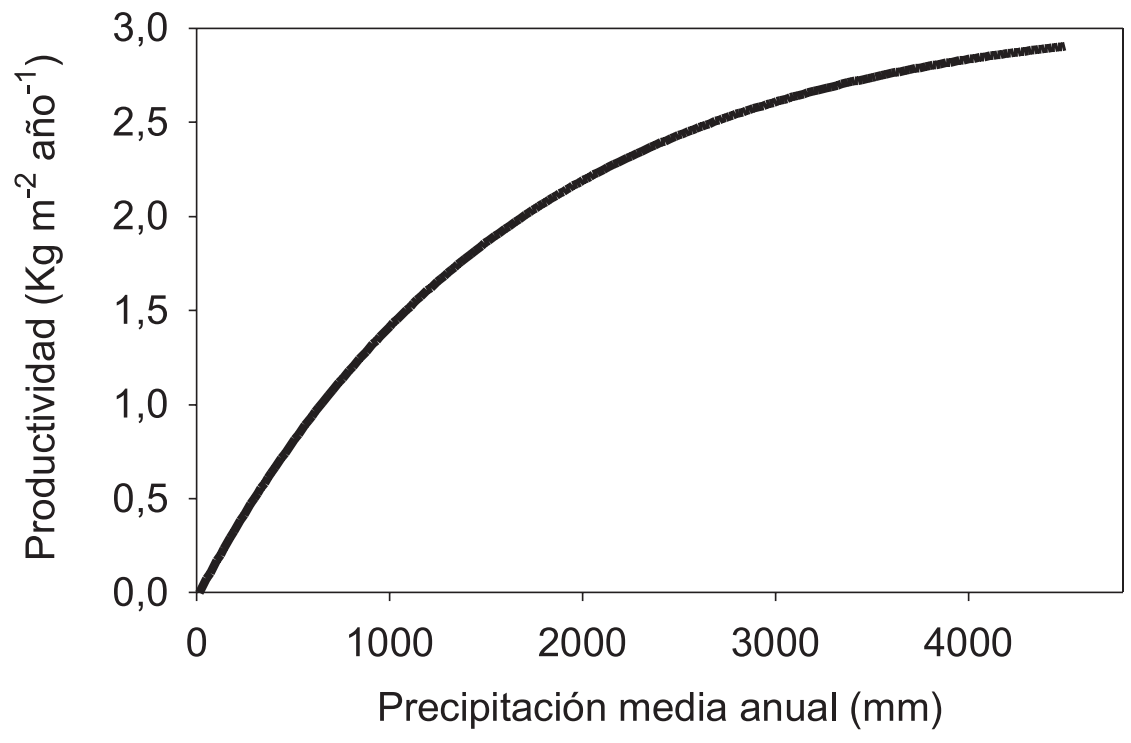

Fuente: modificado a partir de Lieth, 1975

Figura 3b. Rendimiento del cultivo de cebada en función del agua utilizada.

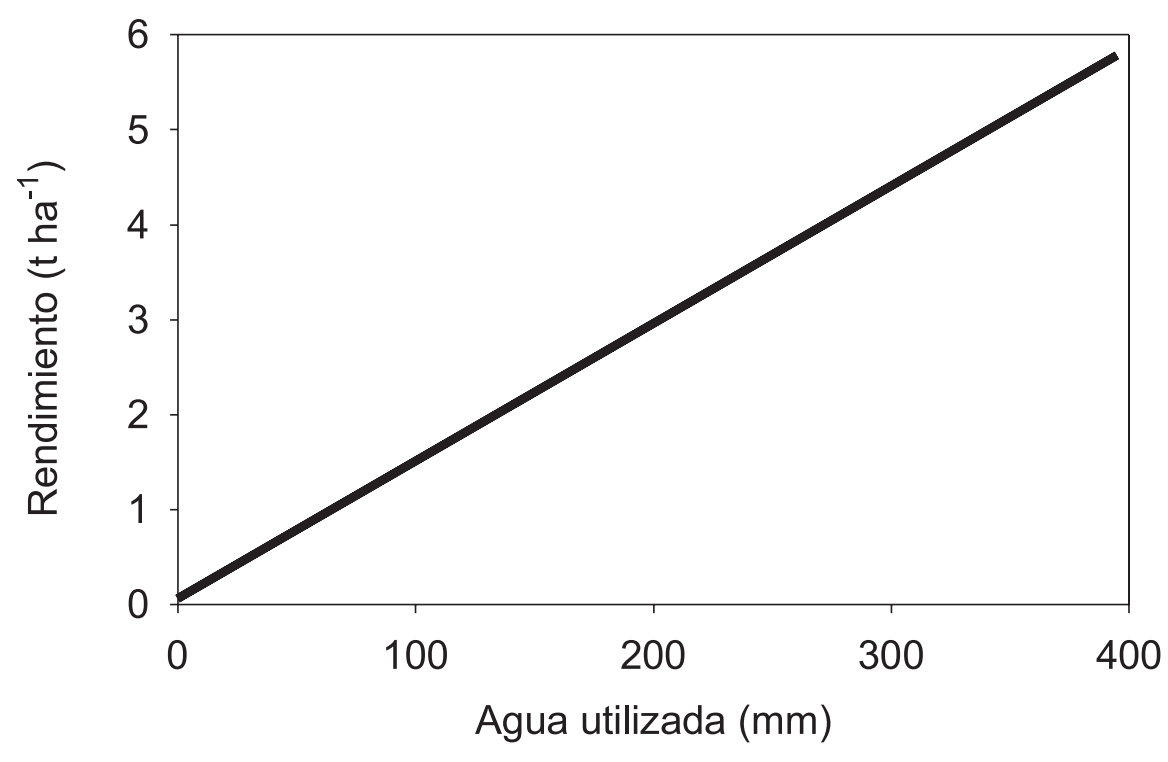

Fuente: modificado a partir de Bacon, 2004 y referencias en su interior. 
para paliar periodos sin ingresos a veces extremadamente largos. Por otra parte, las necesidades de agua, dependen fuertemente de las condiciones climáticas, que se sintetizan en la evaporación potencial, o demanda atmosférica de agua, parámetro que integra los efectos de la cantidad de radiación, humedad, temperatura ambiental y velocidad del viento. Es obvio que un día de junio supone más gasto que un día de enero y que un día promedio de agosto estira de la planta más agua que un día de abril, aunque ambos tengan el mismo número de horas de sol y similar radiación. Por otra parte, la superficie foliar total y la arquitectura de la planta y el cultivo determinan también el gasto real frente a una determinada demanda atmosférica. La planta desarrolla su arquitectura foliar en función de sus características genéticas y de la disponibilidad de recursos durante el crecimiento. Cuantas más hojas hay, mayor es el porte y más abierta la copa, mayor es el gasto de agua. Así, en función de la disponibilidad de agua, las plantas ajustan el tamaño y la cantidad de hojas.

Además de estos controles, los estomas establecen un control fino sobe el gasto de agua, control que experimenta amplias variaciones a lo largo del día en función de la cantidad de luz y de otras variables ambientales, pero sobretodo en función de la disponibilidad de agua en las hojas. Junto a estas variables, el estoma también depende de la actividad fotosintética, que en condiciones limitantes (a causa de falta de luz, temperaturas bajas o infecciones) provoca el cierre estomático independiente de la disponibilidad hídrica. La regulación de la pérdida de agua en los estomas es un típico mecanismo de control múltiple y se establece mediante variaciones de la apertura, que impone mayor o menor restricción a la difusión libre del vapor de agua desde la cavidad subestomática a la atmósfera. Esta regulación es responsable de buena parte de las variaciones en la eficiencia en el uso del agua por las plantas.

\section{Efectos de la sequía en las plantas: control del gasto de agua y ventajas adaptativas}

Las situaciones de déficit hídrico son muy frecuentes en la vida de las plantas, y de hecho, la disponibilidad hídrica resulta ser el primer factor limitante del crecimiento vegetal y del rendimiento de las cosechas en todo el mundo. En la Cuenca Mediterránea, la disponibilidad de agua no es sólo el factor más limitante de la producción agrícola, cultivos, bosques y matorrales están claramente condicionados por la sequía estival típica del Clima Mediterráneo, sino que además es uno de los principales condicionantes del desarrollo humano.

En un medio terrestre en el que el agua no siempre está disponible incluso en los climas más húmedos, han aparecido múltiples adaptaciones y mecanismos a lo largo de la evolución que han permitido a las plantas superar las situaciones de estrés hídrico más o menos intenso. Así, las plantas han desarrollado diferentes respuestas frente al estrés hídrico que en conjunto implican asegurar la supervivencia de la especie, aumentar la disponibilidad de agua y mejorar la eficiencia de su uso (Schulze, 1986). Resulta difícil generalizar en un patrón común la respuesta de las plantas al déficit hídrico, debido a la variedad en duración e intensidad de dicho déficit y a la variedad de modificaciones morfológicas, fisiológicas y de ciclo de vida que existen como caracteres constitutivos de la especie (adaptaciones) y como respuestas a largo (aclimatación) y a corto plazo (regulación). El interés por generalizar los tipos de respuesta ha conducido a establecer «modelos» de comportamiento que describen conjuntos de características que tienden a presentarse a la vez en respuesta a la sequía y que confieren a la especie mayor capacidad de resistir y completar su ciclo bajo esta limitación ambiental. Se describen así tres «estrategias» o modelos de respuesta frente al déficit hídrico que se fundamentan en las respuestas morfológicas y fisiológicas que reducen y/o retrasan la incidencia y los efectos del déficit hídrico: 
1. Escapar de la sequía, estrategia que consiste principalmente en acoplar el ciclo biológico a los meses con mayor disponibilidad de agua.

2. Tolerar la desecación, que consiste en un conjunto de modificaciones fisiológicas que permiten soportar un cierto grado de deshidratación de los tejidos sin reducción drástica de la actividad vital.

3. Evitar la deshidratación, estrategia que supone aumentar la capacidad de aprovisionamiento de agua y limitar su gasto, incrementando la eficiencia en el uso del agua, lo que aumenta la capacidad de permanecer y sobrevivir durante los períodos de sequía.

El déficit hídrico tiene en conjunto importantes consecuencias para la fisiología y la morfología de las plantas provocando, a corto y medio plazo, un descenso del potencial hídrico, del contenido hídrico relativo y de la conductividad hídrica, así como un aumento de la síntesis de ácido abscísico. Estos hechos dan lugar a un descenso de la conductancia estomática y del área foliar total, así como a cambios metabólicos provocados por la disminución de la concentración de agua en las células. En este contexto, las limitaciones de los procesos fotosintéticos provocadas por el déficit hídrico no son únicamente limitaciones a la difusión de $\mathrm{CO}_{2}$ hacia el estroma, sino que también son importantes las limitaciones en las reacciones fotoquímicas, en el Ciclo de Calvin y en el transporte de asimilados.

El déficit hídrico provoca en las hojas una pérdida de turgencia celular que, junto a la acción del ácido abscísico (ABA), cuya síntesis en las raíces aumenta en sequía, son responsables del cierre de los estomas, lo que supone el lógico aumento de la resistencia estomática a la difusión de $\mathrm{CO}_{2}$ hacia el estroma. A pesar de que está ampliamente aceptado que tiene lugar un cierre estomático en respuesta al déficit hídrico, actualmente no hay consenso en la importancia relativa de este hecho, de forma que se encuentran evidencias que tanto sugieren la presencia de limitaciones no estomáticas de la fotosíntesis desde el inicio del déficit hídrico, como otras que sugieren que las limitaciones estomáticas predominan en el inicio del estrés, y las no estomáticas lo hacen en las fases más avanzadas del mismo. En cualquier caso, el cierre estomático tiene también como consecuencias la reducción de la tasa de transpiración y, por tanto, el incremento de la eficiencia en el uso del agua. En las especies esclerófilas mediterráneas, este tipo de respuesta es la que predomina en los primeros momentos del estrés hídrico, durante el inicio de la estación seca.

Como consecuencia del cierre estomático, tiene lugar una disminución de la disponibilidad de $\mathrm{CO}_{2}$ como aceptor último de electrones. En estas circunstancias, se puede producir una acumulación excesiva de poder reductor (NADPH), lo que puede provocar una «sobreacumulación» de los compuestos intermediarios de la cadena de transporte de electrones, provocando un exceso de excitación de los centros de reacción de las clorofilas, que puede causar fotoinhibición y fotooxidación. Esto hace conveniente limitar la captación de energía luminosa y/o disipar el excedente de energía captada en condiciones de déficit hídrico. Uno de los mecanismos conocidos para limitar el porcentaje de energía luminosa interceptada es el cambio de posición de las hojas, de forma que su ángulo de inclinación varía para disminuir la cantidad de luz interceptada. La pérdida total o parcial de hojas durante la estación seca constituye un mecanismo extremo para reducir la captación de energía luminosa, el cual está presente en algunas especies típicamente mediterráneas, como Euphorbia dendroides, en el primer caso, y algunas especies de los géneros Cistus y Phlomis, en el segundo caso. Otros mecanismos que reducen la captación de energía luminosa son la presencia de estructuras reflectoras, tales como pelos, capas de ceras y/o espinas. Las especies mediterráneas, y las de climas áridos y semi-áridos en general, ejemplifican estas adaptaciones, siendo las especies de la familia de las Cactáceas las que presentan una adaptación más clara. La mayoría de especies de cactos han perdido las hojas y sus órganos fotosintéticos son tallos cilíndricos o esféricos con una baja relación superficie/volumen, 
están recubiertos por una gruesa cutícula de ceras y en algunos casos presentan pelos y otras estructuras reflectantes. Todos los mecanismos anteriormente expuestos, además de reducir la cantidad de luz interceptada, disminuyen la pérdida de agua por parte de la planta, constituyendo una adaptación a la falta de agua y el consiguiente exceso de luz que están íntimamente ligados, especialmente en los ecosistemas mediterráneos.

Cuando la energía interceptada es excesiva respecto a la capacidad de utilización, su disipación puede hacerse bien por vía no-fotoquímica, es decir, antes de su incorporación a la cadena de transporte electrónico, o bien por vía fotoquímica, esto es, por mecanismos que consumen el ATP y NADPH sintetizados a partir del transporte electrónico. La disipación térmica asociada al ciclo de de-epoxidación de las xantofilas (ciclo VAZ) constituye el principal mecanismo de disipación no fotoquímico, mientras que la reacción de Mehler, proceso de reducción de $\mathrm{O}_{2}$ por la ferrodoxina en el fotosistema I, y la fotorrespiración constituyen las principales vías fotoquímicas de disipación. Algunos de estos procesos no son, aún hoy, conocidos con detalle, no obstante es destacable la íntima relación que parece existir entre ellos. Así, la de-epoxidación de las xantofilas está condicionada por la presencia de $\Delta \mathrm{pH}$ transtilacoidal, que a su vez es consecuencia de la acumulación de $\mathrm{H}^{+}$en el lumen. Esto sucede cuando hay un mayor consumo de NADPH que de ATP. La fotorrespiración y, especialmente, la reacción de Mehler suponen un mayor consumo de aquél, propiciando así el mantenimiento de $\Delta \mathrm{pH}$ transtilacoidal. Así, estos dos mecanismos, además de contribuir a la disipación de energía por vía fotoquímica, propiciarían la disipación por vía no-fotoquímica mediante el establecimiento de las condiciones necesarias para la activación del ciclo VAZ. El grado de desarrollo de estos mecanismos varía entre especies de forma importante, constituyéndose, en ocasiones, en factores clave de la adaptación de una especie a ambientes estresantes.

Otros procesos que se ven afectados por el déficit hídrico son la fotofosforilación, diversos aspectos del Ciclo de Calvin, como la regeneración de la RubP, o la actividad de la RubisCO, la acumulación de almidón y de azúcares solubles en hojas, el transporte de fotoasimilados hacia las raíces, y la emisión de isoprenos y monoterpenos. No obstante, el grado en que se ven afectados por el déficit hídrico la mayoría de estos procesos aún hoy no está claro, por lo que tampoco lo está la ventaja relativa de las diferentes adaptaciones relacionados con los mismos.

En definitiva, el déficit hídrico, además de provocar ajustes en el crecimiento y en las relaciones hídricas, causa importantes modificaciones en el conjunto de procesos que conducen a la asimilación de $\mathrm{CO}_{2}$. Junto a los efectos directamente relacionados con el cierre estomático, tienen lugar limitaciones no estomáticas que afectan a diversos procesos fotosintéticos. Las consecuencias son, por una parte la reducción del gasto de agua y el incremento de la eficiencia en su uso, y por otra la disminución de la asimilación de $\mathrm{CO}_{2}$, y por tanto del crecimiento y la producción.

\section{Eficiencia del uso del agua: balance coste/beneficio}

Como se ha comentado anteriormente, en el proceso de intercambio de gases, en el que las plantas incorporan a su biomasa (fijan) carbono de la atmósfera también tiene lugar una «pérdida» (evapotranspiración) de vapor de agua desde la planta a la atmósfera. Así, la eficiencia en el uso del agua (EUA) de las plantas puede entenderse de manera genérica como el volumen de agua que éstas necesitan consumir (evapotranspirar) para incorporar a su biomasa una determinada cantidad de carbono proveniente de la atmósfera (en la que se encuentra en forma de $\mathrm{CO}_{2}$ ). De esta manera, la eficiencia en el uso del agua de las plantas dependerá principalmente de dos tipos de factores: en primer lugar, de aquellas características propias de la especie y variedad que tengan relación con la capacidad de optimización 
de los procesos de asimilación de carbono y de evapotranspiración de agua; y en segundo lugar, de las características del ambiente en el que crece y se desarrolla la planta.

Por otra parte, la eficiencia en el uso del agua también dependerá de la escala a la que se considere, pues bajo la sencilla definición que se ha indicado anteriormente, la eficiencia en el uso del agua puede ser considerada a escala de cultivo o de ecosistema, a escala de planta entera y a escala de hoja. Además del nivel de organización considerado, la escala temporal, esto es el período de tiempo que se considera en la medida de la EUA, añade complejidad tanto a la medida como a la interpretación fisiológica y ecológica de este parámetro. A continuación, se tratará la importancia y el significado de cada una de las diferentes maneras de considerar la eficiencia en el uso del agua, dejando para el siguiente apartado los comentarios referentes a la influencia de los factores genéticos y ambientales en la eficiencia en el uso del agua.

La EUA de los cultivos y ecosistemas tan sólo puede ser estimada de una manera aproximada, pues en condiciones de campo resulta muy difícil conocer con exactitud la cantidad de agua que realmente han consumido las plantas y su crecimiento en biomasa acumulada. Así, habitualmente, el consumo de agua se estima a partir de datos indirectos de precipitación (ingreso de agua) y el volumen de agua perdido por escorrentía, percolación o evaporación directa del suelo y que en ningún caso ha sido consumida por las plantas. Por otra parte, otro factor determinante a la hora de medir o estimar la EUA de cultivos y ecosistemas es la forma de medir o estimar la producción de biomasa y, en el caso de los cultivos, qué se considera producción: la biomasa total producida o la parte del cultivo que es cosechada. En cualquier caso, la producción de biomasa radicular habitualmente se ignora por razones metodológicas, siendo en el mejor de los casos estimada. De esta forma, bajo el concepto de la eficiencia en el uso del agua de cultivos y ecosistemas aparecen diversas realidades originadas a partir de diferentes formas de medir y estimar el agua consumida y la biomasa producida (tabla 3). Más allá de las limitaciones metodológicas anteriormente descritas, existe la posibilidad de medir la EUA de cultivos herbáceos homogéneos mediante el uso de lisímetros. Los lisímetros no son más que grandes básculas situadas bajo tierra que pesan una parcela de terreno. Si esta parcela está cubierta por un cultivo herbáceo homogéneo en el que no hay zonas de suelo desnudo, de manera que se minimiza la evaporación directa del suelo, las variaciones de peso se corresponderán, en su mayor parte, a la evapotranspiración de agua del cultivo. Así, los lisímetros constituyen uno de los métodos más exactos de medir la eficiencia en el uso agua a escala de cultivo. No obstante, su elevado coste y el hecho de que tan sólo sean funcionales con cultivos herbáceos homogéneos constituyen importantes limitaciones a su uso.

Tabla 3

ALGUNOS PARÁMETROS UTILIZADOS PARA ESTIMAR LA EFICIENCIA EN EL USO DEL AGUA DE CULTIVOS Y ECOSISTEMAS

\begin{tabular}{|l|l|}
\hline Consumo de agua & Producción de biomasa \\
\hline Evapotranspiración de la masa vegetal & Biomasa aérea producida \\
\hline Transpiración de la masa vegetal & Biomasa fresca de cosecha \\
\hline Precipitación & Biomasa seca de cosecha \\
\hline Precipitación + Riego & Biomasa total estimada** \\
\hline Precipitación corregida* & Equivalentes de glucosa \\
\hline
\end{tabular}

*Precipitación - (escorrentía + percolación + evaporación ${ }_{\text {suelo }}$ )

**Biomasa aérea + biomasa radicular estimada 
La eficiencia en el uso del agua a nivel de planta es un parámetro con un valor principalmente experimental, pues habitualmente se obtiene en condiciones controladas en las que las plantas se encuentran en macetas o en sistemas en los que el agua consumida puede medirse con exactitud. Por este motivo, este parámetro constituye en sí la eficiencia en el uso del agua real, pues sus dos componentes, agua consumida y biomasa producida (incluida la biomasa radicular), pueden ser determinadas con exactitud. Sin embargo, presenta dos inconvenientes: en primer lugar se trata de una medida realizada en condiciones experimentales, por lo que su extrapolación a condiciones reales de campo debe ser llevada a cabo con cautela; en segundo lugar, su medida es muy sencilla pero muy laboriosa debido a que el consumo de agua se realiza por diferencia de peso y la producción de biomasa radicular implica la separación de las raíces del suelo, lo que no siempre es sencillo. A pesar de estos dos inconvenientes, la eficiencia en el uso del agua a nivel de planta entera es un parámetro muy útil en la comparación de especies y variedades.

La medida de la eficiencia en el uso del agua a escala foliar tiene un enorme valor experimental. Uno de los principales motivos para su toma en consideración es la posibilidad de representar un parámetro de medida relativamente sencilla que pueda ser representativo de la EUA a escala de planta entera. Desde este punto de vista, se han desarrollado principalmente dos técnicas para su medida: el intercambio de gases, técnica que generalmente integra un período de tiempo breve o muy breve, y el análisis de la discriminación isotópica del carbono, que integra un período de tiempo mucho mayor.

Las medidas de intercambio de gases en hojas permiten determinar la transpiración (salida de agua de la planta) y la fotosíntesis neta (entrada neta de $\mathrm{CO}_{2}$ en las hojas). La eficiencia en el uso del agua a escala foliar es el cociente de estos dos parámetros, y se considera a dos niveles. En primer lugar, la eficiencia de la transpiración, esto es: asimilación de $\mathrm{CO}_{2}$ / transpiración $\left(\mathrm{A} / \mathrm{E}, \mu \mathrm{mol} \mathrm{CO}_{2} / \mathrm{mol} \mathrm{H}_{2} \mathrm{O}\right)$; en segundo lugar, la eficiencia intrínseca en el uso del agua: asimilación de $\mathrm{CO}_{2} /$ conductancia estomática $\left(\mathrm{A} / \mathrm{g}, \mu \mathrm{mol} \mathrm{CO}_{2} / \mathrm{mol} \mathrm{H}_{2} \mathrm{O}\right)$. Ambas formas de medir la EUA tienen las mismas unidades, pero la diferencia fundamental es que la eficiencia de la transpiración depende de la planta y de las condiciones ambientales, de forma que un mismo grado de apertura estomática puede traducirse en una tasa de transpiración muy diferente si la humedad ambiental varía; mientras que la eficiencia intrínseca mide diferencias relacionadas con la capacidad de la hoja para regular la fotosíntesis y la conductancia estomática, y que son independientes de las condiciones atmosféricas en el momento de la medida. Como se ha apuntado anteriormente, ambos parámetros presentan como principal limitación el hecho de que son medidas instantáneas, y por tanto integran un período muy breve de tiempo, por lo que su extrapolación a la EUA de la planta entera presenta la doble dificultad de relacionar parámetros medidos a escalas organizacional y temporal diferentes.

La estimación de la eficiencia en el uso del agua mediante el análisis de la discriminación isotópica del carbono se basa en la coexistencia de forma natural en la atmósfera de dos isótopos estables de carbono: ${ }^{12} \mathrm{C} \mathrm{y}{ }^{13} \mathrm{C}$. Ambos isótopos presentan características químicas idénticas, pero el hecho de que el ${ }^{13} \mathrm{C}$ sea más pesado que el ${ }^{12} \mathrm{C}$ da lugar a que la tasa de difusión del ${ }^{13} \mathrm{C}$ en la hoja, y la de su asimilación por la Rubisco, son más bajas que la del ${ }^{12} \mathrm{C}$, por lo que la proporción de ${ }^{13} \mathrm{C}$ es menor en los tejidos vegetales que en la atmósfera. Esta proporción varía con la disponibilidad de $\mathrm{CO}_{2}$ en la hoja, y por tanto con la conductancia estomática. De esta forma la discriminación isotópica del ${ }^{13} \mathrm{C}\left(\Delta^{13} \mathrm{C}\right)$ de la hoja está íntimamente ligada con la A/g de esa hoja durante todo el período de formación de la misma. De la misma manera, la composición isotópica del carbono de otros órganos de la planta tales como raíces y tallos ofrece información que puede ser relevante en el estudio de la EUA de diferentes genotipos, no obstante, en estos casos también intervienen 
otros procesos fisiológicos como la partición de fotoasimilados entre los diferentes órganos, por lo que la interpretación de estos datos es a día de hoy más confusa.

Por desgracia, ninguna de las dos técnicas permite obtener resultados que sean fácilmente extrapolables a escala de planta entera, de forma que la relación entre la EUA medida a ambas escalas varía con frecuencia según la especie y las condiciones experimentales. Así, el establecimiento de protocolos sencillos que permitan obtener relaciones claras y estables entre la EUA a escala de planta entera y a escala foliar es una de las cuestiones que más permitirían avanzar en el estudio comparativo de la EUA entre especies y variedades.

\section{Variabilidad ambiental y genética en la eficiencia en el uso del agua por las plantas}

\subsection{Variabilidad ambiental:}

Las variaciones inducidas por las condiciones ambientales en la eficiencia en el uso del agua se conocen desde los primeros experimentos descritos sobre este tema a finales del siglo XVII, y que tratan de los requerimientos hídricos de la menta en función de la fuente de agua, que en definitiva se debía a variaciones en el contenido en nutrientes disueltos. Más tarde, en medidas sistemáticas del requerimiento de agua por los cultivos, la comparación del gasto de agua de la alfalfa entre zonas de clima contrastado en EEUU demostraba que el requerimiento hídrico puede doblarse en función de las diferencias climáticas. Variaciones de este rango, y superiores, se han determinado posteriormente en otros muchos cultivos. El estudio de las causas de estas variaciones condujo a la definición de la evaporación potencial (ETP) como parámetro integrador de las diferentes variables que inciden en la demanda de agua de la atmósfera (Radiación, temperatura, humedad, velocidad del viento), y que hoy resulta imprescindible cuando se trata de comparar valores de EUA.

La comparación, más reciente, de la EUA del tomate en condiciones de cultivo que van desde campo a túnel de plástico, invernadero, o cultivo en atmósfera controlada muestran variaciones mucho más amplias (Tabla 4) que resultan muy ilustrativas del rango de variación de este parámetro y también de las posibilidades de optimización del consumo de agua por las plantas. En vegetación natural, se registran asimismo variaciones importantes en función de la localización, la estación de año y el tipo de suelo.

Tabla 4

REQUERIMIENTOS HÍDRICOS (LITROS DE AGUA POR KILO DE PRODUCTO FRESCO) EN DIFERENTES CONDICIONES AMBIENTALES Y SISTEMAS DE CULTIVO

\begin{tabular}{|l|c|}
\hline & Tomate \\
\hline Israel \& Almeria, campo & 60 \\
\hline Almeria, túnel de plástico (1990) & 40 \\
\hline Israel, Invernadero de vidrio sin calefacción & 30 \\
\hline Almeria, Invernadero de plástico perfeccionado & 27 \\
\hline $\begin{array}{l}\text { Holanda, Invernadero de vidrio con control de condiciones climáticas y } \\
\text { alto CO }\end{array}$ & 22 \\
\hline Holanda, idéntico al anterior reutilizando el agua de drenaje & 15 \\
\hline
\end{tabular}

Fuente: Stanghellini, 2005 y referencias en su interior. 


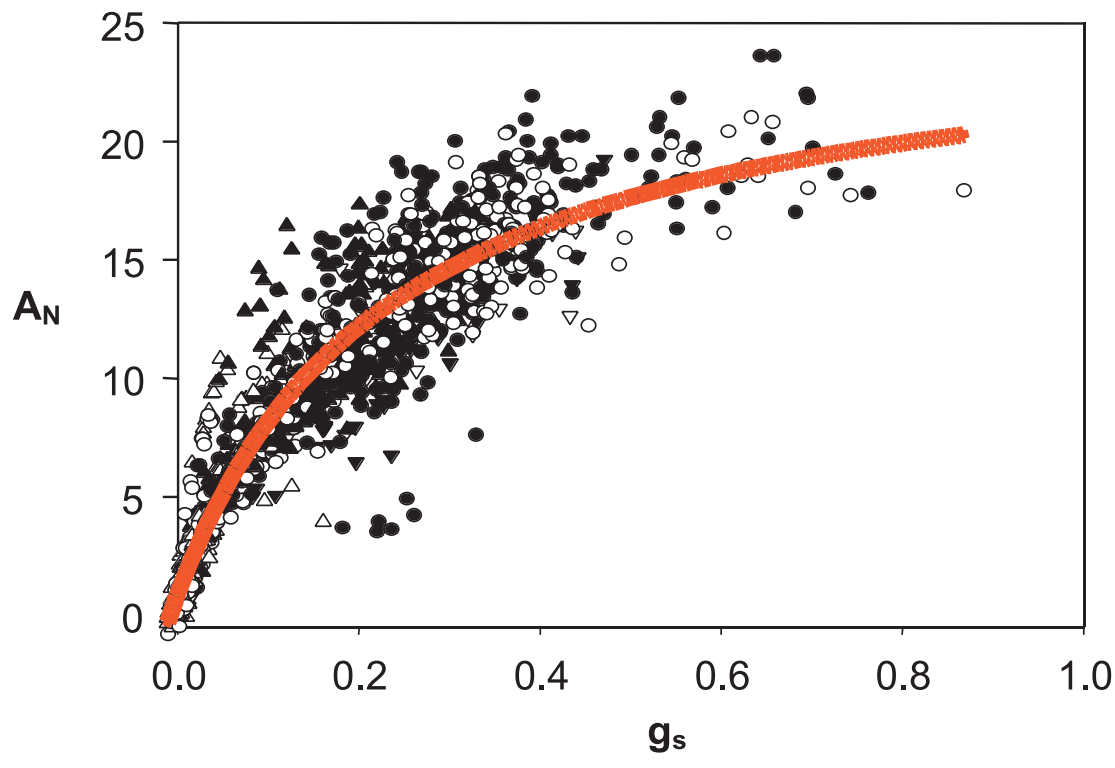

FIgURa 4. Relación entre fotosíntesis neta $\left(\mathrm{A}_{\mathrm{N}} ; \mu \mathrm{mol} \mathrm{CO} \mathrm{CO}^{-2} \mathrm{~s}^{-1}\right)$ y conductancia estomática $\left(\mathrm{g}_{\mathrm{s}} ; \mathrm{mol}\right.$ $\left.\mathrm{H}_{2} \mathrm{O} \mathrm{m}^{-2} \mathrm{~s}^{-1}\right)$ en 23 variedades de vid. Los datos fueron tomados durante 10 años en plantas crecidas en campo y en maceta. La regresión presenta una $R^{2}=0,88(n=1347)$.

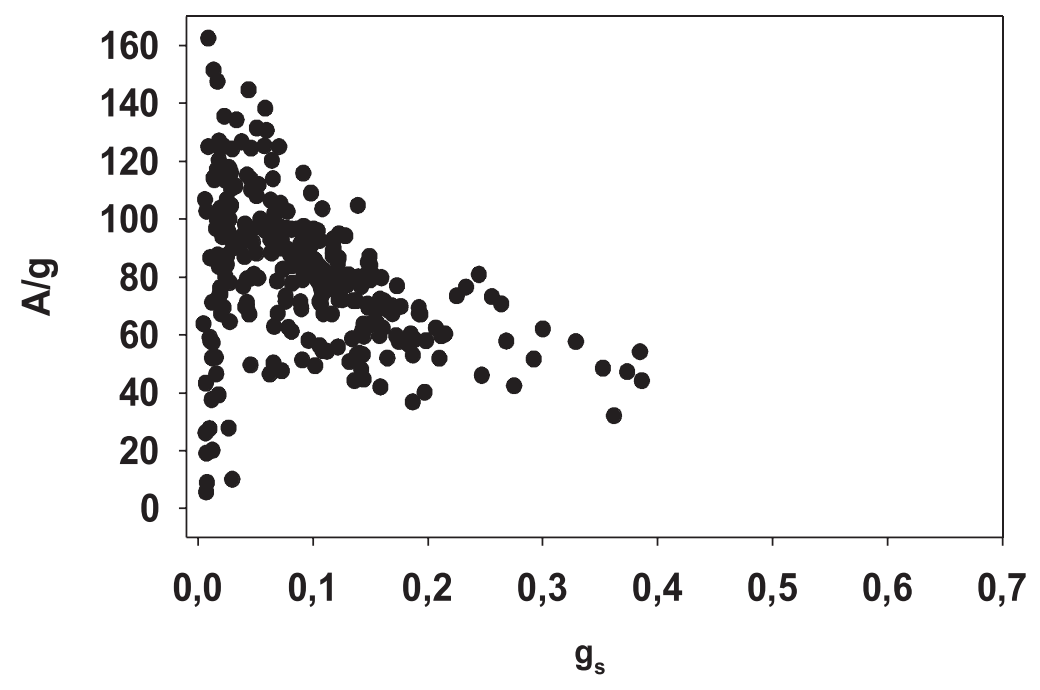

Figura 5. Relación entre eficiencia intrínseca en el uso del agua $\left(\mathrm{A} / \mathrm{g} ; \mu \mathrm{mol} \mathrm{CO}_{2} \mathrm{~mol}^{-1} \mathrm{H}_{2} \mathrm{O}\right)$ y conductancia estomática $\left(\mathrm{g}_{\mathrm{s}} ; \mathrm{mol} \mathrm{H}_{2} \mathrm{O} \mathrm{m}^{-2} \mathrm{~s}^{-1}\right)$ en 23 variedades de vid. Los datos fueron tomados durante 10 años en plantas crecidas en campo y en maceta. 
Dentro de unas determinadas condiciones de cultivo, la tasa de agua transpirada en las hojas depende del gradiente de vapor de agua entre los estomas y el aire, y de lo abierto que estén los estomas: El gradiente se produce entre una superficie húmeda (las paredes celulares de la cámara subesomática) y el aire, y la evaporación requiere energía. A mayor disponibilidad de energía y gradiente, mayor será la tasa de evaporación. La vía para evitar la deshidratación frente a esta imposición del ambiente es el recubrimiento de las hojas con una epidermis poco permeable y el control de la apertura de los estomas, que en buena parte obedece a la disponibilidad de agua en las raíces. Así la planta ajusta su gasto a la disponibilidad hídrica, reduciendo fuertemente (hasta a la décima parte) el agua transpirada. Sin embargo, como se ha comentado anteriormente, el cierre estomático provoca también mayores dificultades de difusión del $\mathrm{CO}_{2}$ hacia los cloroplastos y con ello drásticas reducciones de la fotosíntesis. La relación fotosíntesis/apertura estomática ha sido objeto de amplios estudios, y nuestro equipo ha podido demostrar que dicha relación se mantiene similar en diferentes condiciones ambientales para un amplio número de variedades cultivadas y de especies vegetales (Medrano et al., 2002; Cifre et al., 2005). La relación presenta por tanto una base muy amplia en el mundo vegetal (Figura 4).

En base a esta relación, podemos apreciar que la tasa de fotosíntesis (que corresponde a la producción de nueva biomasa), llega al máximo cuando la conductancia es la mitad o menos que la máxima, o bien que g puede reducirse a un tercio del máximo con reducciones de A inferiores al 10\%. Estos resultados, extrapolados a producción de biomasa significarían que con gastos de agua en torno al 33\% del máximo, la cosecha sólo se reduciría un $10 \%$. Es decir, controlando la disponibilidad de agua en las raíces (que indirectamente regulan la apertura de los estomas) podemos incrementar fuertemente la eficiencia en el uso del agua.

De hecho, si analizamos a nivel de estomas la EUA, en términos de eficiencia intrínseca en el uso del agua (A/g), podemos observar (Figura 5) que para valores muy altos de $\mathrm{g}$ (situación de riego abundante, sin limitaciones hídricas para la planta), A/g es mínimo, y este valor crece exponencialmente según decrece g. Esta curva de respuesta, si la deducimos matemáticamente de la relación A/g (Figura 5) nos ofrecería una relación puramente exponencial, con valores máximos de A/g a valores mínimos de g. Sin embargo, la curva construida con datos reales muestra claramente un máximo para valores muy bajos de $\mathrm{g}, \mathrm{y}$ una fuerte caída posterior. Es decir, en condiciones de cierre estomático severo, la eficiencia en el uso del agua vuelve a ser mínima. $\mathrm{O}$ visto de otra forma, en valores extremos de sequía, las plantas se tornan menos eficientes en el uso del agua. Las bases fisiológicas de esta paradoja han sido una interesante tarea para nuestro equipo (Flexas et al., 2004).

\subsection{Aplicaciones prácticas}

La aplicación de estos conocimientos fisiológicos en campo supone que con un control fino del riego, podemos mantener la planta en condiciones de $\mathrm{g}$ en las que el gasto de agua sea inferior a la mitad del máximo y por tanto la EUA resulte el doble o superior. Es decir, hay grandes oportunidades de mejorar la EUA controlando el riego. En este sentido, mostraremos dos casos prácticos y una perspectiva de futuro.

El primer caso práctico se refiere a experimentos (primavera y verano) realizados en un cultivo hortícola convencional, la lechuga, en condiciones de campo, (Finca Experimental Sa Canova, Sa Pobla, Mallorca) con dos sistemas de riego, goteo y microaspersión, y tres dosis, la control (100\%) corresponde al riego habitual de los horticultores de la zona y dos dosis restringidas en torno al 80 y $60 \%$ del control. En todos los tratamientos, el volumen de riego se ajustó mediante válvulas y se midió con contadores de agua. En el momento 
de recolección, todas las parcelas tenían un aspecto similar. Los pesos y tamaños de las lechugas fueron similares (Tabla 5) y el comprador, tras invitarle a contemplar detenidamente las parcelas, no hizo distinciones. Con volúmenes de riego cercanos a la mitad de lo habitual, el volumen de cosecha y de ingresos obtenidos fue similar.

Tabla 5

GASTO TOTAL DE AGUA (RIEGO + PRECIPITACIÓN) Y TAMAÑO DE LAS LECHUGAS BAJO DIFERENTES DOSIS Y TÉCNICAS DE RIEGO A LO LARGO DE SU CULTIVO. SE REALIZARON DOS EXPERIMENTOS: EL EXPERIMENTO 1 TUVO LUGAR ENTRE ABRIL Y JUNIO Y EL EXPERIMENTO 2 ENTRE MAYO Y JULIO

\begin{tabular}{|c|c|c|c|c|c|c|}
\hline Experimento & \multicolumn{3}{|c|}{ MICROASPERSIÓN } & \multicolumn{3}{c|}{ GOTEO } \\
\hline & Litros & \% riego & $\begin{array}{c}\text { Longitud } \\
\text { lechuga }\end{array}$ & Litros & \% riego & $\begin{array}{c}\text { Longitud } \\
\text { lechuga }\end{array}$ \\
\hline 1 & 50.284 & 100 & 43 & 45.535 & 91 & 42 \\
\hline 1 & 42.720 & 85 & 42 & 35.060 & 70 & 44 \\
\hline 1 & 32.745 & 65 & 39 & 28.498 & 57 & 43 \\
\hline 2 & 52.448 & 100 & 37 & 50.360 & 96 & 34 \\
\hline 2 & 36.730 & 70 & 42 & 38.942 & 74 & 33 \\
\hline 2 & 28.102 & 54 & 38 & 26.706 & 51 & 35 \\
\hline
\end{tabular}

Esto significa que, pasando de los datos fisiológicos a la escala agronómica real, las reducciones del gasto de agua de riego hasta casi la mitad pueden hacerse sin reducciones sensibles de la cosecha, tal como puede predecirse de la figura 4.

La EUA puede por tanto mejorarse notablemente en los sistemas agrícolas. La cuestión clave es la capacidad de control del volumen de riego. Cabe preguntarse porqué los agricultores no reducen las dosis de riego. La respuesta está en que, las irregularidades del terreno, de los sistemas de riego, las pérdidas, etc. aconsejan añadir coeficientes de seguridad a las dosis básicas, a fin de tener cosechas uniformes en todas las parcelas. La mejora de los sistemas de riego, la sectorización y automatización de las instalaciones, en definitiva, la agricultura de la precisión permite incrementar fuertemente la EUA de los cultivos. En este sentido, la introducción de contadores de agua resulta clave para reconocer la capacidad de ahorro.

El siguiente ejemplo práctico se ha realizado en cultivos de vid, en condiciones de campo y en maceta, manejando dosis de riego desde un 100\% de la ETP hasta un 30\% durante las fases de envero y maduración. Los datos en campo, (Finca Can Ribas, Consell, Mallorca), son interesantes en términos de mantenimiento de la producción, pero mucho más en términos de incremento de la calidad del fruto, que en viña es un parámetro clave al fijar los precios y por tanto el rendimiento final. La experiencia en diez años consecutivos en campo, en condiciones de fuerte sequía en general, (suelo pedregoso), muestra que dosis elevadas de riego se corresponden con cosechas más altas pero de muy inferior calidad (Medrano et al., 2003). En dosis limitadas, en torno al 30\% de la ETP, la producción es sensiblemente superior a la sequía manteniendo calidades muy altas. En cultivos leñosos, con raíces extensas, el agua disponible para la planta es difícil de determinar, y experimentos con dosis similares sobre suelos de más calidad, (mayor capacidad de retención de agua) muestran resultados muy diferentes, con reducciones de calidad a dosis muy moderadas de riego. Efectivamente, lo que gobierna el funcionamiento de la planta es el agua dispo- 
nible, que en ciertos casos es suficiente, aunque en superficie aparente estar a mínimos. La consecuencia es que para universalizar la dosis de riego, en cultivos leñosos, en los que queremos incrementar la EUA o la calidad de la cosecha (que suele estar siempre a dosis subóptimas de riego) la ETP resulta una referencia insuficiente.

En consecuencia, nuestro equipo ha tratado de experimentar nuevos indicadores fisiológicos y entre estos, la conductancia estomática resulta especialmente sensible pues refleja muy directamente la disponibilidad de agua para la planta en todo su sistema radicular. Sin embargo, la medida de g, requiere equipamiento muy costoso, personal altamente cualificado y medida directa en campo. Esto limita considerablemente su aplicación directa como sensor del estatus hídrico de la planta. Los medidores de flujo de savia son equipamientos relativamente baratos, de uso menos sofisticado y que pueden medir en continuo, almacenar datos que bien pueden recogerse tras unos días o bien pueden trasmitirse directamente a un ordenador de control de riegos. Esto permite disponer de estimaciones del gasto real de la planta bastante ajustadas, y que en viña presentan una correspondencia muy alta con el gasto real de la planta (Escalona et al, 2003). En grandes plantaciones de frutales y en otras leñosas, los medidores de flujo de savia pueden resultar muy eficaces en el ajuste fino de las dosis y momentos de riego, y contribuir fuertemente a la mejora de la EUA en los cultivos.

Las relaciones de la conductancia estomática con el flujo de savia son obvias a nivel conceptual. El flujo refleja la transpiración y ésta depende directamente de la apertura estomática. Sin embargo, la fuerte influencia de las condiciones ambientales, la disposición de la masa foliar, el estado del cultivo etc. hacen que la correspondencia real no sea tan directa como puede deducirse de la teoría.

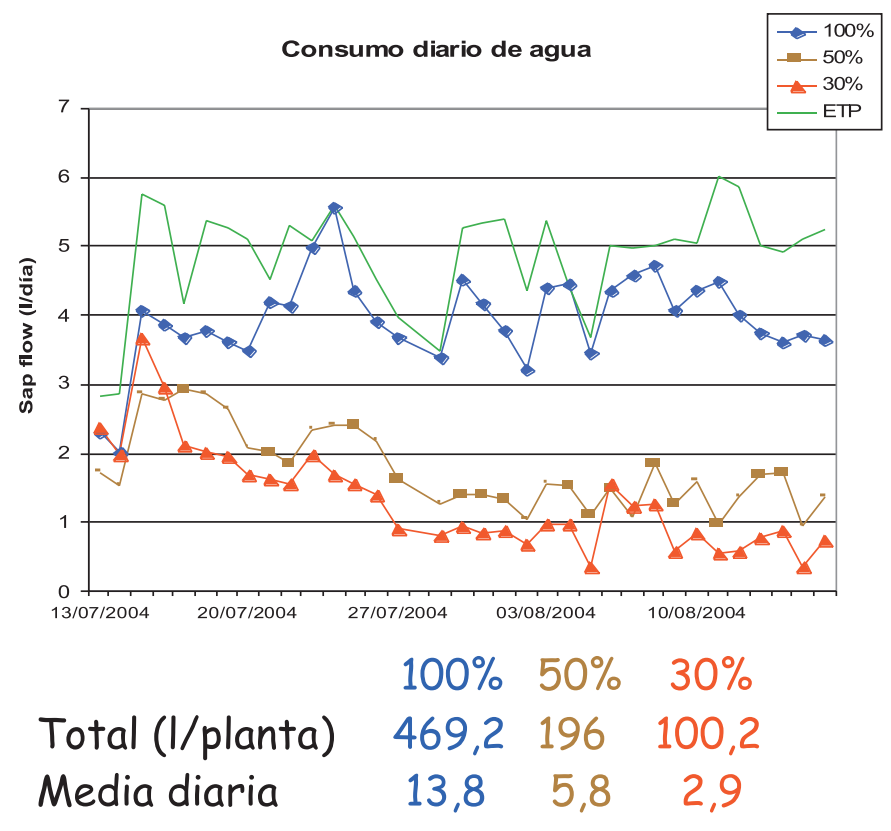

Figura 6. Variación del consumo diario de agua de plantas de vid, variedad Tempranillo, en tres regímenes de riego en condiciones experimentales (plantas crecidas en contenedores de $70 \mathrm{~L}$ en exterior en Mallorca). 
En un experimento reciente en viña, mantenida en contenedores de $70 \mathrm{~L}$ en la que, por tanto, se conoce el agua disponible, durante el envero y maduración se mantuvieron tres niveles de disponibilidad hídrica en base a la determinación de la g en zonas de alto gasto ( $\mathrm{g}>250 \mathrm{mmol} \mathrm{H}_{2} \mathrm{O} \mathrm{m} \mathrm{m}^{-2} \mathrm{~s}^{-1} ; 100 \%$ ), sequía moderada (g entre 150 y 250; 50\%) y sequía severa (g por debajo de $150 ; 30 \%$ ). En paralelo, se midió el gasto real de agua mediante el flujo de savia y la eficiencia intrínseca en el uso del agua (A/g) (figuras 6 y 7). El gasto total se redujo a menos del $25 \%$ mientras que la producción por cepa se mantuvo en un $70 \%$ y la calidad resultó sensiblemente mejor (datos no publicados).

De todo lo anteriormente expuesto cabe resaltar que las condiciones ambientales condicionan fuertemente el gasto de agua de las plantas. El conocimiento más profundo de las bases fisiológicas de la eficiencia en el uso del agua, permite disponer de un marco sobre el que avanzar en nuevos planteamientos de optimización de la EUA. A nivel más experimental, el dominio de las condiciones climáticas mediante invernaderos, o cámaras sofisticadas de atmósfera controlada, supone multiplicar por cuatro la EUA (tabla 4). En condiciones menos controladas, una regulación más fina del gasto de agua de riego, permite doblar y más que doblar la EUA en cultivos hortícolas y leñosos respectivamente. La optimización del uso del agua por las plantas es por tanto teóricamente posible y prácticamente realizable. Nos queda la tarea de investigar y experimentar mucho más ampliamente los rangos y límites de nuestra capacidad de mejora.

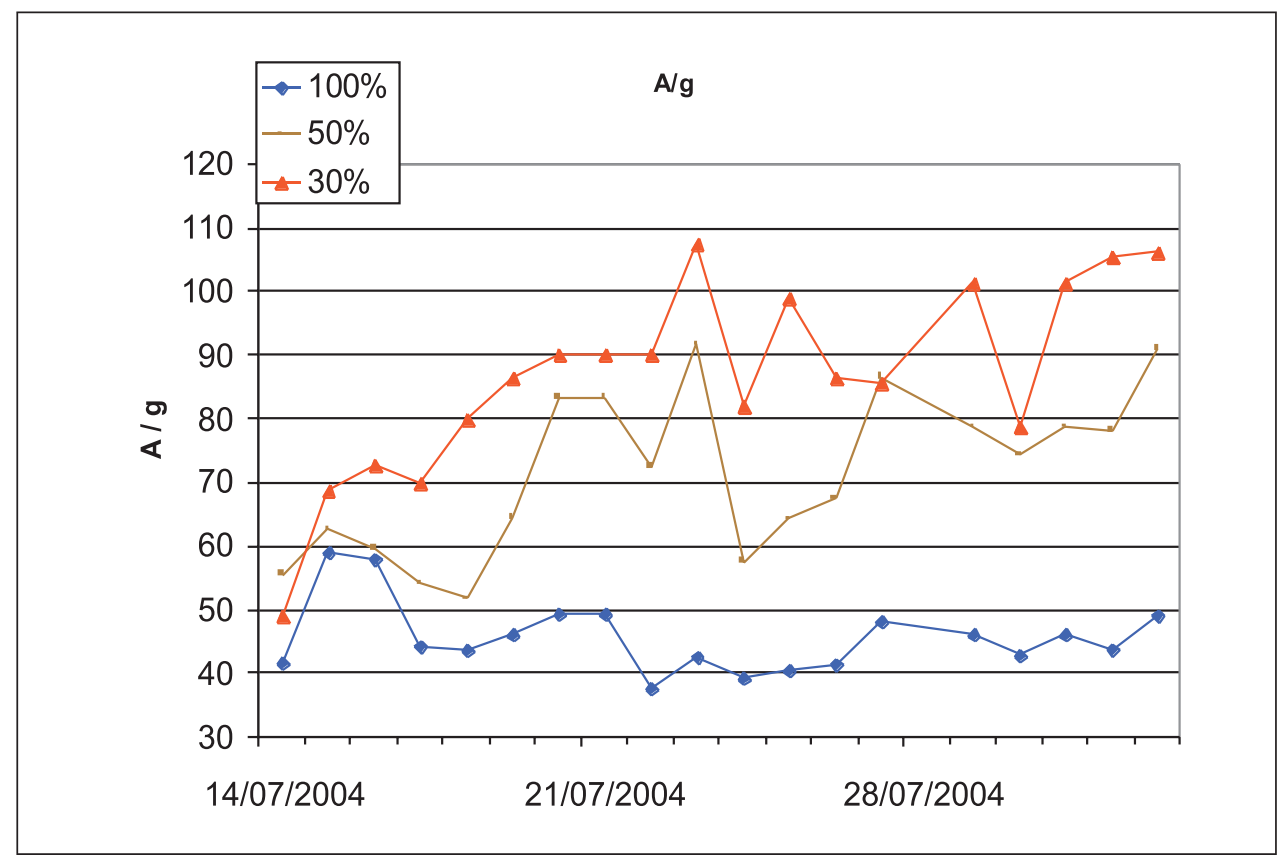

FiguRa 7. Variaciones de la eficiencia intrínseca en el uso del agua en función de la disponibilidad hídrica de plantas de vid, variedad Tempranillo, en tres regímenes de riego en condiciones experimentales (plantas crecidas en contenedores de $70 \mathrm{~L}$ en exterior en Mallorca). 


\subsection{Variabilidad genética}

La existencia de importantes diferencias en la eficiencia en el uso del agua medida a diferentes escalas entre especies y entre variedades de una misma especie permite un cierto margen de actuación a la hora de planificar una explotación agrícola, un jardín o un campo de golf con el ahorro de agua como uno de los criterios fundamentales. De hecho, la EUA es uno de los criterios que se utiliza en la selección de variedades de cultivos de zonas áridas y semi-áridas. A continuación se comentarán algunos ejemplos en los que se ha estudiado la variabilidad Inter e intraespecífica de la EUA a diferentes escalas.

\subsubsection{Variabilidad en la EUA en especies leñosas mediterráneas}

Las especies leñosas mediterráneas, ya sean esclerófilas perennifolias o semi-deciduas, presentan una importante variación estacional en la EUA intrínseca (A/g). En la tabla 6,

Tabla 6

VARIACIÓN DE LA EFICIENCIA INTRÍNSECA EN EL USO DEL AGUA (A/g) Y DE LA CONDUCTANCIA ESTOMÁTICA $(g)$ EN ESPECIES LEÑOSAS MEDITERRÁNEAS. LOS DATOS FUERON TOMADOS EN PLANTAS CRECIDAS EN CONDICIONES DE CAMPO EN LA ISLA DE MALLORCA (datos no publicados)

\begin{tabular}{|c|c|c|c|c|}
\hline Especie & & $\mathbf{n}$ & $\begin{array}{c}\mathrm{A} / \mathrm{g} \\
\left(\mu \mathrm{mol} \mathrm{CO}_{2} \mathrm{~mol} \mathrm{H}_{2} \mathrm{O}^{-1}\right)\end{array}$ & $\begin{array}{c}\mathrm{g} \\
\left(\mathrm{mol} \mathrm{H}_{2} \mathrm{O} \mathrm{m}^{-2} \mathbf{s}^{-1}\right) \\
\end{array}$ \\
\hline \multirow[t]{3}{*}{ Cistus albidus } & $\min$ & & 19.7 & 0.009 \\
\hline & $\max$ & & 102.7 & 0.628 \\
\hline & $\mathrm{m} \pm \mathrm{s} . \mathrm{d}$ & 56 & $60.1 \pm 18.8$ & $0.169 \pm 0.152$ \\
\hline \multirow[t]{3}{*}{ Cistus monpeliensis } & $\min$ & & 35.6 & 0.007 \\
\hline & $\max$ & & 134.9 & 0.528 \\
\hline & $\mathrm{m} \pm \mathrm{s} . \mathrm{d}$ & 65 & $77.9 \pm 24.1$ & $0.182 \pm 0.134$ \\
\hline \multirow{3}{*}{$\begin{array}{r}\text { Olea europaea } \\
\text { sylvestris }\end{array}$} & $\min$ & & 25.1 & 0.005 \\
\hline & $\max$ & & 156.9 & 0.344 \\
\hline & $\mathrm{m} \pm \mathrm{s} . \mathrm{d}$ & 67 & $88.1 \pm 26.3$ & $0.110 \pm 0.072$ \\
\hline \multirow[t]{3}{*}{ Pistacia lentiscus } & $\min$ & & 43.7 & 0.007 \\
\hline & $\max$ & & 125.1 & 0.278 \\
\hline & $\mathrm{m} \pm \mathrm{s} . \mathrm{d}$ & 74 & $81.0 \pm 18.4$ & $0.100 \pm 0.060$ \\
\hline \multirow[t]{3}{*}{ Quercus ilex } & $\min$ & & 37.5 & 0.015 \\
\hline & $\max$ & & 147.6 & \\
\hline & $\mathrm{m} \pm \mathrm{s} . \mathrm{d}$ & 34 & $77.9 \pm 22.2$ & $0.122 \pm 0.061$ \\
\hline \multirow[t]{3}{*}{ Rhamnus alaternus } & $\min$ & & 30.4 & 0.004 \\
\hline & $\max$ & & 144.7 & 0.175 \\
\hline & $\mathrm{m} \pm \mathrm{s} . \mathrm{d}$ & 40 & $82.1 \pm 23.8$ & $0.083 \pm 0.054$ \\
\hline
\end{tabular}


Tabla 7

ASIMILACIÓN NETA DE CO, (A), CONDUCTANCIA ESTOMÁTICA $(\mathrm{g}) \mathrm{Y}$ EFICIENCIA INTRÍNSECA EN EL USO DEL AGUA (A/g) DE 20 GENOTIPOS DE

VID DE LAS ISLAS BALEARES Y 2 GENOTIPOS DE AMPLIA DISTRIBUCIÓN CULTIVADOS EN CONDICIONES DE ABASTECIMIENTO HÍDRICO ÓPTIMO. EN UNA MISMA COLUMNA, LOS DATOS SEGUIDOS DE LETRAS DIFERENTES SON SIGNIFICATIVAMENTE DIFERENTES A $p<0,05$

\begin{tabular}{|c|c|c|c|}
\hline Variedad & $\begin{array}{c}\mathbf{A} \\
(\mu \mathrm{molsCO} \\
\left.\mathbf{m}_{2} \cdot \mathbf{m}^{-2} \cdot \mathbf{s}^{-1}\right)\end{array}$ & $\begin{array}{c}\mathrm{g} \\
\left(\mathrm{mols} \text { de } \mathrm{H}_{2} \mathrm{O} \mathrm{m}^{-2} \mathbf{s}^{-1}\right)\end{array}$ & $\begin{array}{c}\mathrm{A} / \mathrm{g} \\
\left(\mathrm{mmolsCO}_{2} \cdot \mathrm{mols}_{2} \mathrm{O}^{-1}\right)\end{array}$ \\
\hline Aleluya & $8,4 \pm 1,8^{a}$ & $0,174 \pm 0,028^{a}$ & $47,3 \pm 3,4^{\mathrm{abcd}}$ \\
\hline Argamusa & $8,7 \pm 1,6^{a}$ & $0,215 \pm 0,063^{\mathrm{ab}}$ & $44,7 \pm 6,9^{\mathrm{abc}}$ \\
\hline Batista & $11,6 \pm 1,6^{\mathrm{abcd}}$ & $0,226 \pm 0,026^{\mathrm{abc}}$ & $51,1 \pm 1,8^{\mathrm{abcd}}$ \\
\hline Boal & $8,4 \pm 0,7^{\mathrm{a}}$ & $0,209 \pm 0,018^{\mathrm{ab}}$ & $40,7 \pm 4,4^{\mathrm{abc}}$ \\
\hline Callet & $17,5 \pm 0,4^{\mathrm{e}}$ & $0,463 \pm 0,017^{\mathrm{e}}$ & $37,7 \pm 0,6^{\mathrm{a}}$ \\
\hline Calop Blanc & $9,5 \pm 0,9^{\mathrm{a}}$ & $0,138 \pm 0,034^{\mathrm{a}}$ & $73,2 \pm 9,9^{\mathrm{e}}$ \\
\hline Escursach & $13,0 \pm 1,7^{\text {abcde }}$ & $0,209 \pm 0,038^{\mathrm{ab}}$ & $63,2 \pm 2,8^{\mathrm{de}}$ \\
\hline Esperó de gall & $10,8 \pm 1,7^{\mathrm{ab}}$ & $0,219 \pm 0,025^{\mathrm{ab}}$ & $48,7 \pm 2,9^{\text {abcd }}$ \\
\hline Fogoneu & $8,8 \pm 0,8^{\mathrm{a}}$ & $0,158 \pm 0,019^{\mathrm{a}}$ & $56,3 \pm 4,3^{\text {cd }}$ \\
\hline Gargollasa & $11,2 \pm 1,2^{\mathrm{abcd}}$ & $0,203 \pm 0,041^{\mathrm{ab}}$ & $58,0 \pm 7,5^{\text {cde }}$ \\
\hline Giró & $11,7 \pm 1,0^{\mathrm{abcd}}$ & $0,217 \pm 0,041^{\mathrm{ab}}$ & $56,1 \pm 6,4^{\mathrm{bcd}}$ \\
\hline Grumiere & $11,3 \pm 1,5^{\mathrm{abcd}}$ & $0,273 \pm 0,036^{\mathrm{abc}}$ & $41,6 \pm 1,0^{\mathrm{abc}}$ \\
\hline Malvasia & $8,9 \pm 2,4^{\mathrm{a}}$ & $0,184 \pm 0,071^{\mathrm{a}}$ & $52,5 \pm 5,5^{\mathrm{abcd}}$ \\
\hline Mancín & $11,2 \pm 0,8^{\text {abcd }}$ & $0,224 \pm 0,040^{\mathrm{abc}}$ & $52,5 \pm 6,9^{\text {abcd }}$ \\
\hline Manto Negro & $10,7 \pm 1,8^{\mathrm{ab}}$ & $0,257 \pm 0,008^{\mathrm{abc}}$ & $41,5 \pm 6,1^{\mathrm{abc}}$ \\
\hline Mollar & $11,1 \pm 0,9^{\mathrm{abc}}$ & $0,268 \pm 0,017^{\mathrm{abc}}$ & $41,3 \pm 1,6^{\mathrm{abc}}$ \\
\hline Monastrell & $14,5 \pm 1,5^{\text {bcde }}$ & $0,327 \pm 0,029^{\mathrm{bcd}}$ & $44,6 \pm 3,6^{\mathrm{abc}}$ \\
\hline Prensal Blanc & $15,7 \pm 1,1^{\text {cde }}$ & $0,411 \pm 0,019^{\mathrm{de}}$ & $38,5 \pm 3,8^{\mathrm{ab}}$ \\
\hline Quigat & $10,4 \pm 0,6^{\mathrm{ab}}$ & $0,188 \pm 0,013^{\mathrm{a}}$ & $55,7 \pm 2,1^{\mathrm{bcd}}$ \\
\hline Sabater & $16,0 \pm 2,8^{\mathrm{de}}$ & $0,354 \pm 0,098^{\text {cde }}$ & $49,4 \pm 7,7^{\mathrm{abcd}}$ \\
\hline Chardonnay & $12,4 \pm 0,6^{\text {abcd }}$ & $0,267 \pm 0,028^{\mathrm{abc}}$ & $47,1 \pm 4,0^{\text {abcd }}$ \\
\hline Cabernet S. & $11,9 \pm 1,2^{\mathrm{abcd}}$ & $0,190 \pm 0,031^{\mathrm{a}}$ & $63,9 \pm 5,9^{\mathrm{de}}$ \\
\hline
\end{tabular}

Fuente: Bota et al., 2001. 
se muestran valores mínimos, máximos y medios de $\mathrm{A} / \mathrm{g}$ medidos a lo largo de tres años en individuos de diferentes especies mediterráneas, observándose que las variaciones son importantes. Las especies semi-deciduas (Cistus spp.) son las que experimentan una A/g media más baja, lo que, junto con sus valores extremos de conductancia estomática, sugiere que estas especies están menos adaptadas, al menos desde el punto de vista de la eficiencia en el uso del agua, que las especies esclerófilas. No obstante, la persistencia de las especies del género Cistus en los ecosistemas mediterráneas parece estar asegurada gracias a su gran producción de semillas, lo que les permite una elevada tasa de regeneración. Por otra parte, entre las especies esclerófilas, destaca la elevada EUA media del acebuche, lo que sin duda le ayuda a mantener elevadas producciones en ambientes mediterráneos relativamente secos.

\subsubsection{Variabilidad en la EUA en variedades de Vid}

La Vid (Vitis vinifera) es una especie cultivada desde hace muchos siglos, lo que sin duda ha facilitado la existencia de numerosas variedades registradas. Asimismo, y debido a su interés económico, es una de las especies vegetales en la que mayor número de trabajos científicos se han publicado, incluyendo diferentes estudios de la variabilidad en la eficiencia en el uso del agua entre cultivares. En la tabla 7, se muestran valores de eficiencia intrínseca en el uso del agua de 22 genotipos de Vid, observándose una gran variación a pesar de que todas las plantas se encontrasen en un estado hídrico óptimo. Así, variedades como Argamasa, Callet y Prensal Blanc, entre otras, presentan valores de A/g inferiores a $45 \mu$ mols $\mathrm{CO}_{2} \cdot \mathrm{mol} \mathrm{H}_{2} \mathrm{O}^{-1}$, mientras que, en el extremo opuesto, la variedad Calop Blanch presenta una $\mathrm{A} / \mathrm{g}$ de $73,2 \mu \mathrm{mols} \mathrm{CO}_{2} \cdot \mathrm{mol} \mathrm{H}_{2} \mathrm{O}^{-1}$. Más allá de la importante variabilidad observada, lo que permitiría considerar este factor a la hora de seleccionar variedades de Vid para zonas semi-áridas, resulta interesante identificar aquellas variedades que, junto a una elevada EUA, presentan una elevada tasa de asimilación (A). De esta forma, resulta destacable la variedad Escursach que presenta valores relativamente elevados tanto de $\mathrm{A} / \mathrm{g}$ como de A, lo que sugiere que es una variedad con posibilidad de alcanzar elevadas tasas de crecimiento en condiciones de baja disponibilidad hídrica. En el extremo opuesto, se podría identificar a las variedades Boal y Argamasa como genotipos que muestran valores bajos de $\mathrm{A}$ y de $\mathrm{A} / \mathrm{g}$, lo que sugiere un bajo potencial productivo unido a una baja eficiencia en el uso del agua. No obstante, cabe destacar lo comentado anteriormente, la eficiencia en el uso del agua a escala foliar no siempre refleja la EUA a escala de planta o de cultivo, pues en este salto organizacional aparecen muchos otros factores además de los estrictamente fisiológicos.

\subsubsection{Variabilidad en la EUA en variedades de cereales}

Al igual que la Vid, los cereales son especies cultivadas desde hace muchos siglos, por lo que existen, y todavía hoy se desarrollan, numerosas variedades. Uno de los parámetros más importantes a la hora de seleccionar genotipos de diferentes especies de cereales ha sido tradicionalmente la producción, de biomasa y de grano. A partir de estos datos y de una estimación más o menos aproximada del agua consumida, ya sea mediante la estimación de la evapotranspiración, de la precipitación o de algún otro parámetro de los comentados en la tabla 3, se puede estimar la EUA a escala de cultivo.

En la tabla 8, se muestra la variabilidad de EUA en cuatro variedades de trigo a escala de cultivo experimental y su relación con la discriminación isotópica del carbono de tejido foliar $\left(\Delta^{13} \mathrm{C}\right)$, observándose una relación negativa entre ambos parámetros, lo que suele 
ser habitual, pues una mayor concentración de ${ }^{13} \mathrm{C}$ en el tejido foliar suele ser indicativo de una menor disponibilidad de $\mathrm{CO}_{2}$ para la planta, y por tanto de una mayor limitación de la producción. Por otra parte, la variabilidad de la EUA en estas variedades de trigo es bastante importante, teniendo en cuenta que los datos presentados han sido obtenidos en condiciones experimentales óptimas.

Tabla 8

EFICIENCIA EN EL USO DEL AGUA Y DISCRIMINACIÓN ISOTÓPICA DEL CARBONO DE TEJIDO FOLIAR $\left(\Delta^{13} C\right)$ EN CUATRO VARIEDADES DE TRIGO (TRITICUM SP.)

\begin{tabular}{|c|c|c|}
\hline Variedad & EUA $\left(\mathbf{g}\right.$ materia seca/ $\left.\mathbf{K g ~ H}_{2} \mathbf{O}\right)$ & $\Delta^{13} \mathbf{C}(\%$ ) \\
\hline Karchia & 2,46 & 23,46 \\
\hline Shorawaki & 2,88 & 22,83 \\
\hline Pastor & 2.99 & 21,95 \\
\hline Baviacora & 2,45 & 22,93 \\
\hline
\end{tabular}

(Fuente: Shaheen \& Hood-Nowotny, 2005)

\section{Agradecimientos}

Los trabajos realizados por el Grup de Biologia de les plantes en condicions mediterrànies sobre eficiencia en el uso del agua forman parte de los proyectos: PRIB-2004-10144, financiado por el Govern de les Illes Balears, e INCO-PERMED (PL 509140), financiado por la Unión Europea.

\section{Referencias bibliográficas}

BACON, M.A. (2004). Water use efficiency in plant biology. Blackwell publishing, Oxford, UK, 327 págs.

BOTA, J., FLEXAS, J., MEDRANO, H. (2001). «Genetic variability of photosynthesis and water use in Balearic grapevine cultivars», en Annals of Applied Biology, vol. 138, págs. 353-361.

CIFRE, J., BOTA, J., ESCALONA, JM., MEDRANO, H., FLEXAS, J. (2005): «Physiological tools for irrigation scheduling in grapevines: an open gate to improve water use efficiency?», en Agriculture Ecosystems \& Environment, vol. 106, págs. 159-170.

ESCALONA, J.M., FLEXAS, J., BOTA, J., MEDRANO, H. (2003): «Distribution of leaf photosynthesis and transpiration within grapevine canopies under different drought conditions», en Vitis, vol. 42, págs. 57-64.

FLEXAS, J., BOTA, J., LORETO, F., CORNIC, G., SHARKEY, TD. (2004): «Diffusive and metabolic limitations to photosynthesis under drought and salinity in C3 plants», en Plant Biology, vol. 6, págs. 269-279.

LIETH, H. (1975): «Modeling the primary productivity of the world», en Primary productivity of the Biosphere, Springer-Verlag, págs. 237-263.

MEDRANO, H. ESCALONA, JM., BOTA, J., GULIAS, J., FLEXAS, J. (2002): «Regulation of photosynthesis of $\mathrm{C} 3$ plants in response to progressive drought: the interest of stomatal conductance as a reference parameter», en Annals of Botany, vol. 89, págs. 895-905.

MEDRANO H, ESCALONA, J.M., CIFRE. J., BOTA, J. FLEXAS, J. (2003): «A ten year study on the physiology of two Spanish grapevine cultivars under field conditions: effect of water availability from leaf photosynthesis to grape yield and quality», en Functional Plant Biology, vol. 30, págs. 607-619. 
SHAHEEN, R., HOOD-NOWOTNY, C. (2005). «Effect of drought and salinity on carbon isotope discrimination in wheat cultivars», en Plant Science, vol. 168, págs. 901-909.

SHANTZ, H.L., PIEMEISEL, L.N. (1927): «The water requirements at Akron, Colorado», en Journal of Agricultural Research, vol. 34, págs 1093-1190.

STANGHELLINI, C. (2005): «Irrigation water: use, efficiency and economics», en Improvement in water use efficiency in protected crops, Junta de Andalucía, Sevilla, págs. 23-33.

WORLD RESOURCES INSTITUTE (1994): World resources 1994-95, A guide to the global environment. Oxford University Press, UK. 